\title{
ON THE STRUCTURE OF FINITE GROUPS ISOSPECTRAL TO FINITE SIMPLE GROUPS
}

\author{
MARIYA A. GRECHKOSEEVA \\ Sobolev Institute of Mathematics and Novosibirsk State University, \\ Ac. Koptyuga 4, Novosibirsk, 630090, Russia. \\ ANDREY V. VASIL'EV \\ Sobolev Institute of Mathematics and Novosibirsk State University, \\ Ac. Koptyuga 4, Novosibirsk, 630090, Russia.
}

\begin{abstract}
Finite groups are said to be isospectral if they have the same sets of element orders. A finite nonabelian simple group $L$ is said to be almost recognizable by spectrum if every finite group isospectral to $L$ is an almost simple group with socle isomorphic to $L$. It is known that all finite simple sporadic, alternating and exceptional groups of Lie type, except $J_{2}, A_{6}, A_{10}$ and ${ }^{3} D_{4}(2)$, are almost recognizable by spectrum. The present paper is the final step in the proof of the following conjecture due to V.D. Mazurov: there exists a positive integer $d_{0}$ such that every finite simple classical group of dimension larger than $d_{0}$ is almost recognizable by spectrum. Namely, we prove that a nonabelian composition factor of a finite group isospectral to a finite simple symplectic or orthogonal group $L$ of dimension at least 10 , is either isomorphic to $L$ or not a group of Lie type in the same characteristic as $L$, and combining this result with earlier work, we deduce that Mazurov's conjecture holds with $d_{0}=60$.
\end{abstract}

Keywords. Simple group, symplectic group, orthogonal group, element orders, spectrum of a group.

2010 MSC. 20D06, 20D60.

\section{INTRODUCTION}

The spectrum $\omega(G)$ of a finite group $G$ is the set of element orders of $G$. Groups are isospectral if they have the same spectra. Given a finite group $G$ with nontrivial soluble radical, one can construct infinitely many different finite groups isospectral to $G$ [25, 33]. In contrast, there is a conjecture due to V.D. Mazurov that in general the set of groups isospectral to a finite nonabelian simple group $L$ is finite and consists of groups closely related to $L$. More precisely, Mazurov conjectured 11 that if $L$ is an alternating group of sufficiently large degree or a simple group of Lie type of sufficiently large Lie rank, and $G$ is

E-mail addresses: gma@math.nsc.ru, vasand@math.nsc.ru.

The work is supported by the Russian Foundation for Basic Research (project 13-01-00505).

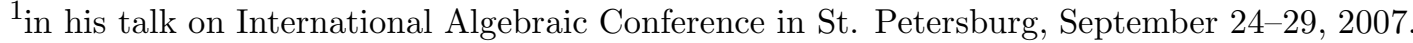


a group isospectral to $L$, then $G$ is an almost simple group with socle isomorphic to $L$. For brevity we refer to a nonabelian simple group $L$ such that every finite group isospectral to $L$ is an almost simple group with socle isomorphic to $L$, as almost recognizable by spectrum.

Gorshkov 12 proved that all alternating groups of degree at least 11 are almost recognizable by spectrum, and recently Vasil'ev and Staroletov [42] completed the investigation of almost recognizability of exceptional groups. Thus Mazurov's conjecture has been reduced to the following: there exists a positive integer $d_{0}$ such that every simple classical group of dimension larger than $d_{0}$ is almost recognizable. The main purpose of the present paper is to prove this conjecture with $d_{0}=60$. As a result, we establish the following theorem (our notation for nonabelian simple groups follows [7]).

Theorem 1. Let $L$ be one of the following nonabelian simple groups:

(i) a sporadic group other than $J_{2}$;

(ii) an alternating group $A_{n}$, where $n \neq 6,10$;

(iii) an exceptional group of Lie type other than ${ }^{3} D_{4}(2)$;

(iv) $L_{n}(q)$, where $n \geqslant 45$ or $q$ is even;

(v) $U_{n}(q)$, where $n \geqslant 45$, or $q$ is even and $(n, q) \neq(4,2),(5,2)$;

(vi) $S_{2 n}(q), O_{2 n+1}(q)$, where either $q$ is odd and $n \geqslant 28$, or $q$ is even and $n \geqslant 20$;

(vii) $O_{2 n}^{+}(q)$, where either $q$ is odd and $n \geqslant 31$, or $q$ is even and $n \geqslant 20$;

(viii) $O_{2 n}^{-}(q)$, where either $q$ is odd and $n \geqslant 30$, or $q$ is even and $n \geqslant 20$.

Then every finite group isospectral to $L$ is isomorphic to some group $G$ with $L \leqslant G \leqslant$ Aut $L$. In particular, there are only finitely many pairwise nonisomorphic finite groups isospectral to $L$.

Theorem 1 is undoubtedly a sum of efforts by numerous mathematicians, and a comprehensive list of references covering its proof is too long to be given here. But for every series of simple groups mentioned in Theorem 1, we cite the work in which the proof for this series was completed, and as a rule this work includes a survey of previous investigations. Thus, see already mentioned [12] and [42] for alternating and exceptional groups. See [29] for sporadic groups, [37, Theorem 1] for linear and unitary groups in odd characteristic, and [40] and [14, Corollary 2] for linear and unitary groups in characteristic 2. The present paper is concerned with symplectic and orthogonal groups.

By [37, Theorem 2 and Proposition 6], if $L$ is a finite simple symplectic or orthogonal group as in (vi) - viii) of Theorem 1 and $G$ is a finite group with $\omega(G)=\omega(L)$, then $G$ has only one nonabelian composition factor and this factor $S$ is a symplectic or orthogonal group having the same underlying characteristic as $L$. If $S \simeq L$, then the soluble radical of $G$ is trivial [15, Theorem 1.1], and hence $G$ is an almost simple group with socle isomorphic to $L$, as required. If $S \nsucceq L L$, then by [41, Theorem 3] there are at most two possibilities for $S$ (see Lemma 3.1 below). We eliminate these possibilities by the following theorem, and thus Theorem 1 follows.

Theorem 2. Let $q$ be a power of a prime $p, L$ one of the groups $S_{2 n}(q)$, where $n \geqslant 2$ and $(n, q) \notin\{(2,2),(2,3)\}, O_{2 n+1}(q)$, where $n \geqslant 3$, or $O_{2 n}^{ \pm}(q)$, where $n \geqslant 4$, and let $G$ be a finite group with $\omega(G)=\omega(L)$. Suppose that some nonabelian composition factor $S$ of $G$ is a group of Lie type over a field of characteristic $p$. Then either $S \simeq L$ or one of the following holds:

(i) $L=S_{4}(q)$, where $q \neq 3^{2 k+1}$, and $S=L_{2}\left(q^{2}\right)$; 
(ii) $L \in\left\{O_{9}(q), S_{8}(q)\right\}$ and $S=O_{8}^{-}(q)$;

(iii) $\{L, G\}=\left\{O_{8}^{+}(2), S_{6}(2)\right\}$;

(iv) $\{L, G\}=\left\{O_{8}^{+}(3), O_{7}(3)\right\}$.

We conclude the introduction with a discussion of nonabelian simple groups that are genuinely not almost recognizable by spectrum. The groups $J_{2}, A_{6}, A_{10},{ }^{3} D_{4}(2), U_{4}(2) \simeq S_{4}(3)$, and $U_{5}(2)$ are not almost recognizable by spectrum; moreover, each of them is isospectral to a group with nontrivial soluble radical (see [3, 25, 27, 29]). All the groups $L_{2}(q), L_{3}(q)$ and $U_{3}(q)$, except for $L_{2}(9), L_{3}(3), U_{3}(5)$ and $U_{3}(p)$ where $p$ is a Mersenne prime and $p^{2}-p+1$ is a prime too, are almost recognizable (see [4], [30,46] and [30,47] respectively). The question is still open for $L_{4}(q)$ and $U_{4}(q)$ with $q$ odd: the group $L_{4}\left(13^{24}\right)$ was proved to be not almost recognizable, and it was conjectured that there are infinitely many $q$ such that $L_{4}(q)$ is not almost recognizable [48. Thus, the exceptions in (ii)-(iii) of Theorem 1 are necessary, while the condition $n \geqslant 45$ in (iv) and ( $\mathrm{v}$ ) can probably be replaced by $n \geqslant 5$.

The simple group $S_{4}(q)$ is almost recognizable if and only if $q=3^{m}$ with $m>1$ odd; moreover, the case (ii) of Theorem 2 is possible [26, 30]. It is known that $\omega\left(O_{8}^{+}(2)\right)=\omega\left(S_{6}(2)\right)$ and $\omega\left(O_{8}^{+}(3)\right)=\omega\left(O_{7}(3)\right)$ and there are no other finite groups with such spectra [24, 34. The group $S_{8}(2)$ is not almost recognizable [28]. In the last section we generalize this result to all groups $S_{8}(q)$ with $q$ even, showing the case (iii) of Theorem 2 is possible when $q$ is even. We suspect that it is also possible when $q$ is odd (and this is proved for $L=O_{9}(q)$ in [17]). Thus it seems likely that all the exceptions appearing in Theorem 2 can be realised. On the other hand, we believe that all other symplectic and orthogonal groups are almost recognizable by spectrum.

To summarize, we propose the following

Conjecture 1. Let $L$ be one of the following groups:

(i) $L_{n}(q)$, where $n \geqslant 5$;

(ii) $U_{n}(q)$, where $n \geqslant 5$ and $(n, q) \neq(5,2)$;

(iii) $S_{2 n}(q)$, where $n \geqslant 3, n \neq 4$ and $(n, q) \neq(3,2)$;

(iv) $O_{2 n+1}(q)$, where $q$ is odd, $n \geqslant 3, n \neq 4$ and $(n, q) \neq(3,3)$;

(v) $O_{2 n}^{\varepsilon}(q)$, where $n \geqslant 4$ and $(n, q, \varepsilon) \neq(4,2,+),(4,3,+)$.

Then every finite group isospectral to $L$ is isomorphic to some group $G$ with $L \leqslant G \leqslant$ Aut $L$.

In fact, to prove Conjecture 1 it suffices to show that the only nonabelian composition factor of a group isospectral to a simple classical group $L$ under consideration is not a group of Lie type whose underlying characteristic differs from that of $L$. The existing generic proof of this assertion recently obtained in [37] requires that the dimension of $L$ is as large as stated in Theorem 1. It is worth also mentioning that the assertion was proved in some special cases, in particular for many classical groups with disconnected prime graph (see $[2,8,9,16,19,20,23,32,39]$ for recent research in this area).

\section{Preliminaries}

By $\left(a_{1}, a_{2}, \ldots, a_{k}\right)$ and $\left[a_{1}, a_{2}, \ldots, a_{k}\right]$ we denote respectively the greatest common divisor and least common multiple of positive integers $a_{1}, a_{2}, \ldots, a_{k}$. If $a$ is a positive integer and $r$ is a prime then $\pi(a)$ denotes the set of prime divisors of $a$ and $(a)_{r}$ denotes the highest power of $r$ that divides $a$. 
Lemma 2.1 (Zsigmondy [49]). Let $q \geqslant 2$ and $n \geqslant 3$ be integers with $(q, n) \neq(2,6)$. There exists a prime $r$ such that $r$ divides $q^{n}-1$ but does not divide $q^{i}-1$ for $i<n$.

With notation of Lemma 2.1, we call a prime $r$ a primitive prime divisor of $q^{n}-1$ and denote it by $r_{n}(q)$. By $R_{n}(q)$ we denote the set of all primitive prime divisors of $q^{n}-1$. Observe that $R_{n}(q) \subseteq R_{n}\left(q^{k}\right)$ if $k$ is coprime to $n$, and $R_{n k}(q) \subseteq R_{n}\left(q^{k}\right)$ for all $n$ and $k$.

Given a group $G$, we set $\pi(G)=\pi(|G|)$ and define the prime graph $G K(G)$ as follows: its vertex set is $\pi(G)$ and two different primes $r$ and $s$ are adjacent if and only if $G$ has an element of order rs. We use standard graph-theoretic terminology: a coclique of a graph is a set of pairwise nonadjacent vertices; a neighbourhood of a vertex $v$ of a graph is the subgraph consisting of all vertices adjacent to $v$ and all edges connecting two such vertices.

Lemma 2.2. Let $L$ be a finite nonabelian simple group of Lie type other than $L_{3}(3), U_{3}(3)$, $S_{4}(3) \simeq U_{4}(2)$ and let $G$ be a finite group with $\omega(G)=\omega(L)$. Then the following hold.

(i) There is a nonabelian simple group $S$ such that $S \leqslant \bar{G}=G / K \leqslant$ Aut $S$, where $K$ is the soluble radical of $G$.

(ii) If $\rho$ is a coclique of size at least 3 in $G K(G)$, then at most one prime of $\rho$ divides $|K| \cdot|\bar{G} / S|$.

(iii) If $r \in \pi(G)$ is not adjacent to 2 in $G K(G)$, then $r$ is coprime to $|K| \cdot|\bar{G} / S|$.

Proof. If there is a coclique of size 3 in $G K(L)$, then the assertion is the main theorem of [36] supplemented with [38] and [43, Theorem 7.1]. If there are no cocliques of size 3 in $G K(L)$, then we have that $G K(L)$ is disconnected by [22,44,45]. Then the Gruenberg-Kegel theorem [45, Theorem A] implies that either (i) and (iii) holds true for $G$, or $G$ is a Frobenius or 2-Frobenius group ( $G$ is called 2-Frobenius if $G=A B C$, where $A, A B$ are normal in $G$, $B$ is normal in $B C$, and $A B$ and $B C$ are Frobenius groups). Simple groups of Lie type that can be isospectral to a Frobenius or 2-Frobenius group are described in [1], and these groups are precisely $L_{3}(3), U_{3}(3)$ and $S_{4}(3) \simeq U_{4}(2)$.

We say that a finite group $H$ is a (proper) cover of a finite group $G$ if there is a (nontrivial) normal subgroup $K$ of $H$ such that $H / K \simeq G$.

Lemma 2.3 ( [15, Lemma 2.3]). Let $A$ and $B$ be finite groups. The following are equivalent.

(i) $\omega(H) \nsubseteq \omega(B)$ for any proper cover $H$ of $A$;

(ii) $\omega(H) \nsubseteq \omega(B)$ for any split extension $H=K: A$, where $K$ is a nontrivial elementary abelian group.

Lemma 2.4 ( [24, Lemma 1]). Let $G$ be a finite group, $K$ a normal subgroup of $G$ and $G / K$ a Frobenius group with kernel $N$ and cyclic complement $C$. If $(|N|,|K|)=1$ and $N$ is not contained in $K C_{G}(K) / K$, then $r|C| \in \omega(G)$ for some $r \in \pi(K)$.

Lemma 2.5 ( [15, Lemma 2.7]). Let $S$ be a finite simple group of Lie type over a field of characteristic $p$ and let $S$ act faithfully on a vector space $V$ over a field of characteristic $r$, where $r \neq p$. Let $H=V \rtimes S$ be a natural semidirect product of $V$ by $S$. Suppose that $s$ is a power of $r$ and some proper parabolic subgroup $P$ of $S$ contains an element of order $s$. If the unipotent radical of $P$ is abelian, or both $p$ and $r$ are odd, or $p=2$ and $r$ is not a Fermat prime, or $r=2$ and $p$ is not a Mersenne prime, then $r s \in \omega(H)$. 
We conclude with several lemmas on spectra of symplectic and orthogonal groups. If $p \in \pi(G)$, then $\omega_{p^{\prime}}(G)$ denotes the subset of $\omega(G)$ consisting of numbers coprime to $p$. In Lemmas [2.6 and 2.7, \pm in $\left[a_{1} \pm 1, \ldots, a_{s} \pm 1\right]$ means that we can choose + or - for every entry independently. In Lemma 2.9 , for brevity, we write $\varepsilon$ instead of $\varepsilon 1$ for $\varepsilon \in\{+,-\}$.

Lemma 2.6 ( [5, Corollaries 2 and 6]). Let L be one of the simple groups $S_{2 n}(q)$ or $O_{2 n+1}(q)$, where $n \geqslant 2$ and $q$ is a power of an odd prime $p$. Let $d=1$ if $L=S_{2 n}(q)$ or $n=2$, and let $d=2$ if $L=O_{2 n+1}(q)$ with $n \geqslant 3$. Then $\omega(L)$ consists of all divisors of the following numbers:

(i) $\left(q^{n} \pm 1\right) / 2$;

(ii) $\left[q^{n_{1}} \pm 1, \ldots, q^{n_{s}} \pm 1\right]$, where $s \geqslant 2, n_{i}>0$ for all $1 \leqslant i \leqslant s$ and $n_{1}+\cdots+n_{s}=n$;

(iii) $p^{k}\left(q^{n_{1}} \pm 1\right) / d$, where $k, n_{1}>0$ and $p^{k-1}+1+2 n_{1}=2 n$;

(iv) $p^{k}\left[q^{n_{1}} \pm 1, \ldots, q^{n_{s}} \pm 1\right]$, where $k>0, s \geqslant 2, n_{i}>0$ for all $1 \leqslant i \leqslant s$ and $p^{k-1}+1+$ $2\left(n_{1}+\cdots+n_{s}\right)=2 n$

(v) $p^{k}$ if $2 n=p^{k-1}+1$ for some $k>0$.

Lemma 2.7 ( [5, Corollary 3]). Let $L=S_{2 n}(q)$, where $n \geqslant 2$ and $q$ is even. Then $\omega(L)$ consists of all divisors of the following numbers:

(i) $\left[q^{n_{1}} \pm 1, \ldots, q^{n_{s}} \pm 1\right]$, where $s \geqslant 1, n_{i}>0$ for all $1 \leqslant i \leqslant s$ and $n_{1}+\cdots+n_{s}=n$;

(ii) $2\left[q^{n_{1}} \pm 1, \ldots, q^{n_{s}} \pm 1\right]$, where $s \geqslant 1, n_{i}>0$ for all $1 \leqslant i \leqslant s$ and $n_{1}+\cdots+n_{s}=n-1$;

(iii) $2^{k}\left[q^{n_{1}} \pm 1, \ldots, q^{n_{s}} \pm 1\right]$, where $k \geqslant 2, s \geqslant 1, n_{i}>0$ for all $1 \leqslant i \leqslant s$ and $2^{k-2}+1+$ $n_{1}+\cdots+n_{s}=n$

(iv) $2^{k}$ if $n=2^{k-2}+1$ for some $k \geqslant 2$.

Lemma 2.8 ( [43, Proposition 3.1(5)]). Let $L=O_{2 n}^{ \pm}(q)$, where $n \geqslant 4$ and $q$ is a power of a prime $p$. If $r \in \pi(L) \cap R_{k}(q)$, where $k$ is odd and $k>n-2$, or $k$ is even and $k / 2>n-2$, then $r p \notin \omega(L)$.

Lemma 2.9. Let $L=O_{2 n}^{\varepsilon}(q)$, where $n \geqslant 4, \varepsilon \in\{+,-\}$ and $q$ is a power of a prime $p$. Then $\omega_{p^{\prime}}(L)$ consists of all divisors of the following numbers:

(i) $\left(q^{n}-\varepsilon\right) /\left(4, q^{n}-\varepsilon\right)$;

(ii) $\left[q^{n_{1}}-\delta, q^{n_{2}}-\varepsilon \delta\right] / d$, where $\delta \in\{+,-\}, n_{1}, n_{2}>0, n_{1}+n_{2}=n$; $d=2$ if $\left(4, q^{n}-\varepsilon\right)=4$, $\left(q^{n_{1}}-\delta\right)_{2}=\left(q^{n_{2}}-\varepsilon \delta\right)_{2}$, and $d=1$ otherwise;

(iii) $\left[q^{n_{1}}-\delta_{1}, q^{n_{2}}-\delta_{2}, \ldots, q^{n_{s}}-\delta_{s}\right]$, where $s \geqslant 3, \delta_{i} \in\{+,-\}, n_{i}>0$ for all $1 \leqslant i \leqslant s$, $n_{1}+\cdots+n_{s}=n$ and $\delta_{1} \delta_{2} \ldots \delta_{s}=\varepsilon$.

Proof. The assertion follows, for example, from [6, Theorem 6].

Lemma 2.10. Let $L=O_{8}^{+}(q)$, where $q$ is a power of a prime $p$. Then $\omega(L)$ consists of all divisors of the following numbers:

(i) $\left(q^{4}-1\right) /(2, q-1)^{2},\left(q^{3} \pm 1\right) /(2, q-1), q^{2}-1, p\left(q^{2} \pm 1\right) /(2, q-1)$;

(ii) $p^{2}(q \pm 1) /(2, q-1)$ if $p=2,3$;

(iii) 25 if $p=5$;

(iv) 8 if $p=2$.

Proof. The assertion follows from [5, Corollaries 4 and 9].

Lemma 2.11. Let $L=O_{8}^{-}(q)$, where $q$ is even. Then $\omega(L)$ consists of all divisors of the following numbers: $q^{4} \pm 1,\left(q^{2} \pm q+1\right)\left(q^{2}-1\right), 2\left(q^{2}+1\right)(q \pm 1), 4\left(q^{2}-1\right)$, and 8 . 
Proof. The assertion follows from [5, Corollary 4].

Lemma 2.12. Let $q$ be a power of a prime $p$.

(i) Let $q$ be odd and $n \geqslant 3$. Let $r=r_{2 n-2}(q)$ if $q^{n-1} \equiv 1(\bmod 4)$ and $r=r_{n-1}(q)$ if $q^{n-1} \equiv-1(\bmod 4)$. Then $2 p r \in \omega\left(S_{2 n}(q)\right) \backslash \omega\left(O_{2 n+1}(q)\right)$.

(ii) If $n \geqslant 4$ and $(n, q) \neq(4,2)$, then $\operatorname{pr}_{2 n-2}(q) \in \omega\left(O_{2 n+1}(q)\right) \backslash \omega\left(O_{2 n}^{-}(q)\right)$. If $n \geqslant 4$ is even, then $\operatorname{pr}_{n-1}(q) \in \omega\left(O_{2 n+1}(q)\right) \backslash \omega\left(O_{2 n}^{-}(q)\right)$.

(iii) If $q>3$, then $\left(q^{4}-1\right) /(2, q-1)^{2} \in \omega\left(O_{8}^{+}(q)\right) \backslash \omega\left(S_{6}(q)\right)$.

(iv) If $q$ is odd, then $p\left(q^{2}+1\right) \in \omega\left(S_{6}(q)\right) \backslash \omega\left(O_{8}^{+}(q)\right)$.

(v) $\omega\left(O_{2 n+1}(q)\right) \subseteq \omega\left(S_{2 n}(q)\right)$ for all $n \geqslant 2$.

(vi) $\omega\left(O_{2 n-1}(q)\right) \subseteq \omega\left(O_{2 n}^{ \pm}(q)\right) \subseteq \omega\left(O_{2 n+1}(q)\right)$ for all $n \geqslant 3$.

Proof. (iil) Let $q^{n-1} \equiv \varepsilon(\bmod 4)$. Then $2 p r$ divides $p\left(q^{n-1}+\varepsilon\right)$, and in particular it belongs to $\omega\left(S_{2 n}(q)\right)$ by Lemma 2.6. Suppose that $2 p r \in \omega\left(O_{2 n+1}(q)\right)$. It follows by Lemma 2.6 that $2 p r$ divides either $p^{k}\left(q^{n_{1}} \pm 1\right) / 2$ for some $k \geqslant 1$ and $n_{1}>0$ with $p^{k-1}+1+2 n_{1}=2 n$, or $p^{k}\left[q^{n_{1}} \pm 1, \ldots, q^{n_{s}} \pm 1\right]$ for some $k \geqslant 1, s \geqslant 2, n_{1}, \ldots, n_{s}>0$ with $p^{k-1}+1+2 n_{1}+\cdots+2 n_{s}=2 n$. In fact, by the definition of primitive divisor, it cannot divide a number of the latter form since all $n_{1}, \ldots, n_{s}$ are less than $n-1$. And if $2 p r$ divides $p^{k}\left(q^{n_{1}}+\tau\right) / 2$ with $p^{k-1}+1+2 n_{1}=2 n$ and $\tau= \pm 1$, then by the definition of primitive divisor we have that $k=1, n_{1}=n-1$ and $\tau=\varepsilon$. But then $\left(q^{n_{1}}+\tau\right) / 2$ is odd, a contradiction.

(iii) See [43, Proposition 3.1].

(iii) Let $a=\left(q^{4}-1\right) /(2, q-1)^{2}$. Lemma 2.10 implies that $a \in \omega\left(O_{8}^{+}(q)\right)$. By Lemmas 2.6 and 2.7, the orders of semisimple elements of $S_{6}(q)$ are precisely divisors of $\left(q^{3} \pm 1\right) /(2, q-1)$, $\left(q^{2}+1\right)(q \pm 1) /(2, q-1)$ and $q^{2}-1$. It is clear that $a$ divides none of $\left(q^{3} \pm 1\right) /(2, q-1)$ and $q^{2}-1$. Furthermore,

$$
a=\left(q^{2}+1\right) \cdot \frac{(q+1)}{(2, q-1)} \cdot \frac{(q-1)}{(2, q-1)} .
$$

Since $q>2$, both $(q-1) /(2, q-1)$ and $(q+1) /(2, q-1)$ are greater than one, and so $a$ does not divide $\left(q^{2}+1\right)(q \pm 1) /(2, q-1)$ either.

(iv) -(䜣) See Lemmas 2.6 and 2.10.

(vi) It is well known that $O_{2 n-1}(q)<O_{2 n}^{ \pm}(q)<O_{2 n+1}(q)$, and the assertion follows.

Lemma 2.13. Let $q$ be even, $S=S_{6}(q)$ and $L=O_{8}^{+}(q)$. Suppose that $V$ is a nontrivial $S$-module over a field of characteristic 2 and $H$ is a natural semidirect product of $V$ and $S$. Then $\omega(H) \nsubseteq \omega(L)$.

Proof. In the proof of Lemma 4.1 in [15], it was established that $\omega(H)$ contains at least one of the numbers 16,24 , and $2\left(q^{2}+q+1\right)$. By Lemma 2.10, none of these numbers belong to $\omega(L)$.

\section{Proof of Theorem 2}

As we mentioned in the Introduction, the starting point for our proof of Theorem 2 is the following assertion.

Lemma 3.1. Let $q$ be a power of a prime $p, L$ one of the groups $S_{2 n}(q)$, where $n \geqslant 2$ and $(n, q) \notin\{(2,2),(2,3)\}, O_{2 n+1}(q)$, where $n \geqslant 3$, or $O_{2 n}^{ \pm}(q)$, where $n \geqslant 4$, and let $G$ be a finite group with $\omega(G)=\omega(L)$. Suppose that some nonabelian composition factor $S$ of $G$ is a group of Lie type over a field of characteristic $p$. If $S \not L$, then one of the following holds: 
(i) $L=S_{4}(q)$ and $S=L_{2}\left(q^{2}\right)$;

(ii) $\{L, S\} \subseteq\left\{S_{6}(q), O_{7}(q), O_{8}^{+}(q)\right\}$;

(iii) $\{L, S\} \subseteq\left\{S_{2 n}(q), O_{2 n+1}(q), O_{2 n}^{-}(q)\right\}$ and $n \geqslant 4$;

(iv) $L=O_{2 n}^{+}(q), S \in\left\{S_{2 n-2}(q), O_{2 n-1}(q)\right\}$, and $n \geqslant 6$ is even.

Proof. The assertion is a combination of [41, Theorem 3] and [35, Theorem 1].

Let $q=p^{m}$ and let $L$ be one of the groups $S_{2 n}(q)$, where $n \geqslant 2$ and $(n, q) \notin\{(2,2),(2,3)\}$, $O_{2 n+1}(q)$, where $n \geqslant 3$, or $O_{2 n}^{ \pm}(q)$, where $n \geqslant 4$. Let $G$ be a finite group with $\omega(G)=\omega(L)$. By Lemma 2.2, we have

$$
S \leqslant G / K \leqslant \operatorname{Aut} S
$$

where $K$ is the soluble radical of $G$ and $S$ is a nonabelian simple group. Suppose that $S$ is a group of Lie type over a field of characteristic $p$. By Lemma 3.1, either $S \simeq L$, or $L$ and $S$ are as in the conclusion of Lemma 3.1.

Let $L=S_{4}(q)$, where $q>3$. If $q=3^{m}$ and $m$ is odd, then $G \simeq L$ by [26], and so $S \simeq L$. If $q \neq 3^{m}$ with $m$ odd, then $S=L_{2}\left(q^{2}\right)$ by Lemma 3.1, and this is (ii) of the conclusion of Theorem 2, To examine the other cases, we need some auxiliary results.

Lemma 3.2. Let $L \neq S_{4}(q)$. If $k>2$ and $r \in R_{m k}(p)$, then $r \notin \pi($ Out $S)$.

Proof. Since $S$ is a symplectic or orthogonal group over a field of order $q=p^{m}$, it follows that $\pi($ Out $S) \subseteq\{2,3\} \cup \pi(m)$. By Fermat's Little Theorem $m k$ divides $r-1$ and so $r>3$ and $r>m$.

Lemma 3.3. If $L=S_{2 n}(q)$, where $n \geqslant 3$ and $q$ is odd, then $S \neq O_{2 n+1}(q)$.

Proof. Assume the contrary. It follows by [15. Proposition 1.3] that $K=1$ in this case, and hence $S \leqslant G \leqslant$ Aut $S$. Let $r=r_{2(n-1) m}(p)$ if $q^{n-1} \equiv 1(\bmod 4)$ and $r=r_{(n-1) m}(p)$ otherwise. By Lemma 2.12, there is an element $g$ of order $2 p r$ in $G \backslash S$. By Lemma 3.2, we have $r \notin \pi(G / S)$.

Suppose that $p \in \pi(G / S)$. Then $G$ contains a field automorphism of $S$ of order $p$. By [11, Proposition 4.9.1(a)], the centralizer of this automorphism in $S$ includes $O_{2 n+1}\left(q_{0}\right)$, where $q=q_{0}^{p}$. Therefore $\operatorname{pr}_{2 n}\left(q_{0}\right)$ and $p\left[q_{0}+1, q_{0}^{n-1} \pm 1\right]$ lie in $\omega(G)$. If $p$ does not divide $n$, then $r_{2 n}\left(q_{0}\right) \in R_{2 n}(q)$ and so $p r_{2 n}\left(q_{0}\right) \notin \omega(L)$ by [43, Proposition 3.1]. Let $p$ divide $n$. Then $r_{2 n-2}\left(q_{0}\right) \in R_{2 n-2}(q)$. By the definition of primitive divisor and Lemma 2.6, it follows that $p\left(q_{0}+1\right) r_{2 n-2}\left(q_{0}\right) \in \omega(L)$ if and only if $q_{0}+1$ divides $q^{n-1}+1$, which implies that $n$ is even. But then $q_{0}+1$ does not divide $q^{n-1}-1$, and by the same reasoning we conclude that $p\left(q_{0}+1\right) r_{n-1}\left(q_{0}\right) \notin \omega(L)$. In any case, $\omega(G) \nsubseteq \omega(L)$, a contradiction.

Thus $p, r \notin \pi(G / S)$, and it follows that $g^{p r} \notin S$ and $g^{2} \in S$. Therefore $G \backslash S$ contains an involution $t=g^{p r}$ such that $p r \in \omega\left(C_{S}(t)\right)$. Suppose that $t \in \operatorname{Inndiag} S$. Then Inndiag $S \leqslant G$ and since Inndiag $S \simeq S O_{2 n+1}(q)$, it follows that $q^{n}+1 \in \omega(G) \backslash \omega(L)$, which is impossible. Therefore, by [11, Proposition 4.9.1(d)], we have that $t$ is a field automorphism, and hence $m$ is even. In particular $q \equiv 1(\bmod 4)$ and $r=r_{2(n-1) m}(p)$. By [11, Prop 4.9.1(a,b)], the centralizer $C_{S}(t)$ can be embedded into $S O_{2 n+1}\left(q_{0}\right)$, where $q=q_{0}^{2}$, and so $C_{S}(t)$ has no elements of order $r$, a contradiction.

Lemma 3.4. Suppose $\pi(G)$ contains four different primes $p, s, r$, and $w$ with the following properties: 
(i) S contains a Frobenius subgroup $F$ whose kernel is a p-group and whose complement has order $s$;

(ii) $r \in \pi(K)$;

(iii) $\{s, r, w\}$ is a coclique in $G K(G)$.

Then $p \in \pi(K)$ and $p t \in \omega(G)$ for every $t \in \pi(S) \backslash\{p\}$.

Proof. Since $\{s, r, w\}$ is a coclique in $G K(G)$ and $r \in \pi(K)$, it follows by Lemma 2.2(ii) that $s \notin \pi(K)$. Construct a normal $r$-series of $K$ as follows:

$$
1=R_{0} \leqslant K_{1} \leqslant R_{1} \leqslant \ldots \leqslant K_{l-1} \leqslant R_{l-1} \leqslant K_{l} \leqslant R_{l}=K,
$$

where $K_{i} / R_{i-1}=O_{r^{\prime}}\left(K / R_{i-1}\right)$ and $R_{i} / K_{i}=O_{r}\left(K / K_{i}\right)$ for $1 \leqslant i \leqslant l$.

Suppose first that $K / K_{l} \neq 1$ and let $\tilde{K}=G / K_{l}$ and $\widetilde{G}=G / K_{l}$. Since the group $C_{\widetilde{G}}(\tilde{K}) \tilde{K} / \tilde{K}$ is a normal subgroup of $\tilde{G} / \tilde{K} \simeq G / K$, it either contains $S$ or is trivial. In the former case $C_{\widetilde{G}}(\tilde{K})$ has an element of order $s$, and so $r s \in \omega(G)$, contrary to (iii). In the latter case we apply Lemma 2.4 to the Frobenius group $F$, and again obtain $r s \in \omega(G)$.

Now suppose that $K=K_{l}$ and let $\tilde{R}=R_{l-1} / K_{l-1}, \tilde{K}=K / K_{l-1}$, and $\tilde{G}=G / K_{l-1}$. Since $O_{r^{\prime}}(\tilde{K})=1$, it follows by [18, Lemma 1.2.3] that $C_{\tilde{K}}(\tilde{R}) \leqslant \tilde{R}$. Furthermore, we may assume that $C_{\widetilde{G}}(\tilde{R}) \leqslant \tilde{K}$ as above, and therefore $C_{\widetilde{G}}(\tilde{R}) \leqslant \tilde{R}$. If $p$ does not divide $|\tilde{K}|$, then $(|\tilde{K}|,|F|)=1$ and the Schur-Zassenhaus theorem implies that $\tilde{G}$ has a subgroup isomorphic to $F$. In this case we apply Lemma 2.4 and deduce that $r s \in \omega(G)$, a contradiction. Thus $p$ divides $|\tilde{K}|$. Let $\tilde{P}$ be a Sylow $p$-subgroup of $\tilde{K}, \tilde{Z}=Z(\tilde{P})$ and $\tilde{N}=N_{\tilde{G}}(\tilde{P})$. By the Frattini argument, $\tilde{G} / \tilde{K}=\tilde{N} \tilde{K} / \tilde{K}$, and therefore $\tilde{N}$ has an element $g$ of order $s$. Furthermore, since $C_{\tilde{N}}(\tilde{Z})$ is normal in $\tilde{N}$, this group either is contained in $\tilde{K}$ or has $S$ as a section. In the former case $g$ does not centralize $\tilde{Z}$, and then $\tilde{Z}=[\tilde{Z},\langle g\rangle] \times C_{\tilde{Z}}(g)$ yields $[\tilde{Z},\langle g\rangle] \neq 1$. Thus $[\tilde{Z},\langle g\rangle] \rtimes\langle g\rangle$ is a Frobenius group that acts on $\tilde{R}$ faithfully, and applying Lemma 2.4 once again, we have $r s \in \omega(G)$, a contradiction. Thus $C_{\tilde{N}}(\tilde{Z})$ has $S$ as a section, and so $p t \in \omega(G)$ for every $t \in \pi(S) \backslash\{p\}$, as required.

Lemma 3.5. Let $L=S_{2 n}(q)$ or $L=O_{2 n+1}(q)$, where $n \geqslant 5$. Suppose that $r \in \pi(L) \cap R_{k}(q)$ with $k>2, b \in \omega_{p^{\prime}}(L)$, and $r$ divides $b$.

(i) If $k=2 n$ then $b$ divides $\left(q^{n}+1\right) /(2, q-1)$.

(ii) If $k=2 n-2$ then $b$ divides $\left[q^{n-1}+1, q \pm 1\right]$.

(iii) If $n$ is even and $k=n-1$ then $b$ divides $\left[q^{n-1}-1, q \pm 1\right]$.

Proof. The assertion follows from the definition of primitive prime divisor and Lemmas 2.6 and 2.7 .

Lemma 3.6. Let $L=O_{2 n}^{+}(q)$, where $n \geqslant 6$ is even. Suppose that $r \in \pi(L) \cap R_{k}(q)$ with $k>2, b \in \omega_{p^{\prime}}(L)$, and $r$ divides $b$.

(i) If $k=2 n-2$ then $b$ divides $q^{n-1}+1$.

(ii) If $k=n-1$ then $b$ divides $q^{n-1}-1$.

Proof. By the definition of primitive prime divisor and Lemma 2.9, it follows that $b$ divides $\left[q^{n-1}+1, q+1\right]=q^{n-1}+1$ in (i) and $\left[q^{n-1}-1, q-1\right]=q^{n-1}-1$ in (ii).

Now we return to the proof of Theorem 2 , 
Lemma 3.7. If $L, S \in\left\{S_{6}(q), O_{7}(q), O_{8}^{+}(q)\right\}$, then either $S \simeq L$, or $\{L, G\}=\left\{O_{8}^{+}(2), S_{6}(2)\right\}$, or $\{L, G\}=\left\{O_{8}^{+}(3), O_{7}(3)\right\}$.

Proof. Assume that $S \not L L$. If $q=2$ or $q=3$, then respectively $\{L, G\}=\left\{S_{6}(2), O_{8}^{+}(2)\right\}$ or $\{L, G\}=\left\{O_{7}(3), O_{8}^{+}(3)\right\}$ by [26, 34].

Let $q>3$. Since $\omega(S) \subseteq \omega(L)$, it follows from Lemma 2.12(iii,v) that $S \neq O_{8}^{+}(q)$. Furthermore, if $q$ is odd then $S \neq S_{6}(q)$ by Lemma 2.12(i,iv). Also by Lemma 3.3, if $L=S_{6}(q)$, then $S \neq O_{7}(q)$. Thus $L=O_{8}^{+}(q)$ and $S=O_{7}(q)$ (if $q$ is even we write $O_{7}(q)$ instead of $\left.S_{6}(q)\right)$. In particular, $\pi(S)=\pi(L)$.

By Lemmas 2.6, 2.7, and 2.10, the orders of semisimple elements of $L$ and $S$ are precisely the divisors of

$$
\left(q^{4}-1\right) /(2, q-1)^{2},\left(q^{3} \pm 1\right) /(2, q-1), q^{2}-1
$$

and

$$
\left(q^{2}+1\right)(q \pm 1) /(2, q-1),\left(q^{3} \pm 1\right) /(2, q-1), q^{2}-1
$$

respectively. In particular, if $\operatorname{rr}_{6}(q) \in \omega(L)$ for some $r \in \pi(L)$, then $r$ divides $\left(q^{3}+1\right) /(2, q-$ $1)$, and if $\operatorname{rr}_{3}(q) \in \omega(L)$ for some $r \in \pi(L)$, then $r$ divides $\left(q^{3}-1\right) /(2, q-1)$. Since $\left(q^{3}+1\right) /(2, q-1)$ and $\left(q^{3}-1\right) /(2, q-1)$ are coprime, it follows that $r_{6}(q)$ and $r_{3}(q)$ are not adjacent and have disjoint neighbourhoods in $G K(L)$. Furthermore, $\operatorname{pr}_{6}(q), \operatorname{pr}_{3}(q) \notin \omega(L)$ by Lemma 2.8 ,

We claim that $K \neq 1$ yields $\omega(G) \nsubseteq \omega(L)$. By Lemma 2.3 we may assume that $K$ is an elementary abelian $r$-group for some prime $r$ and $G$ has a subgroup that is isomorphic to a semidirect product of $K$ and $S$. Also we may assume that $S$ acts on $K$ faithfully. Otherwise $S$ centralizes $K$, and hence all primes of $\pi(S)=\pi(L)=\pi(G)$ other than $r$ are adjacent to $r$ in $G K(G)$, contrary to the fact that $r_{3}(q)$ and $r_{6}(q)$ have disjoint neighbourhoods in $G K(L)$.

Let $r \neq p$. Since $r_{3}(q)$ divides the order of a proper parabolic subgroup of $S$ with Levi factor of type $A_{2}$ and $p r_{3}(q) \notin \omega(S)$, the group $S$ has a Frobenius subgroup whose kernel is a $p$-group and whose complement has order $r_{3}(q)$. Furthermore, $S$ has a Frobenius subgroup with kernel of order $q^{2}$ and cyclic complement of order $\left(q^{2}-1\right) /(2, q-1)$ by [13, Lemma 5]. Applying Lemma 2.4, we conclude that $G$ has elements of orders $\operatorname{rr}_{3}(q)$ and $r\left(q^{2}-1\right) /(2, q-1)$. If $r\left(q^{2}-1\right) /(2, q-1) \in \omega(L)$, then keeping in mind that $q>3$ and consulting (3.1), we see that either $r$ divides $\left(q^{2}+1\right) /(2, q-1)$ or $r=2$. In the former case $\operatorname{rr}_{3}(q) \notin \omega(L)$. Let $r=2$. Then the highest power of 2 in $\omega(L)$ is equal to $\left(q^{2}-1\right)_{2}$. Let us consider a parabolic subgroup $P$ of $S O_{7}(q)$ with Levi factor of type $B_{2}$. The Levi factor of $P$ is $A \times B$ where $A \simeq G L_{1}(q), B \simeq S O_{5}(q)$ and both $A$ and $B$ contain elements with spinor norm a non-square (see [21, p. 98]). Since $S O_{5}(q)$ has elements of order $q^{2}-1$, it follows that $P \cap S$ also has elements of order $q^{2}-1$. Furthermore, the Chevalley commutator formula implies that the unipotent radical of $P$ is abelian (see, for example, [31, Lemma 2.2]). Applying Lemma 2.5 we obtain that $2\left(q^{2}-1\right)_{2} \in \omega(G) \backslash \omega(L)$.

Now let $r=p$. If $p=2$ then we apply Lemma 2.13. If $p$ is odd then $p r_{3}(q) \in \omega(G)$ by [15, Lemma 3.2], and so $\omega(G) \nsubseteq \omega(L)$.

Thus $K=1$ and hence $S \leqslant G \leqslant$ Aut $S$. Consulting (3.1) and (3.2), we see that $r_{4 m}(p)\left(q^{2}-\right.$ $1) /(2, q-1)$ lies in $\omega(G) \backslash \omega(S)$. Since $r_{4 m}(p) \notin \pi(G / S)$ by Lemma 3.2, it follows from (3.2) that at least one of the numbers $(q+1) /(2, q-1)$ and $(q-1) /(2, q-1)$ belongs to $\omega(G / S)$. The group Out $S$ is a direct product of Outdiag $S$ of order $(2, q-1)$ and a cyclic group of order $m$. 
If $q$ is odd then $q^{3}+1 \in \omega($ Inndiag $S) \backslash \omega(L)$, and so $G \cap$ Inndiag $S=S$ for both even and odd $q$. Therefore the exponent of $G / S$ divides $m$. However $(q \pm 1) /(2, q-1)=\left(p^{m} \pm 1\right) /(2, p-1)>m$ for $q>3$, a contradiction.

Lemma 3.8. If $L, S \in\left\{S_{2 n}(q), O_{2 n+1}(q), O_{2 n}^{-}(q)\right\}$, where $n \geqslant 4$, then either $S \simeq L$, or $L \in\left\{O_{9}(q), S_{8}(q)\right\}$ and $S=O_{8}^{-}(q)$.

Proof. Assume that $S \neq L$. Since $\omega(S) \subseteq \omega(L)$, it follows by Lemma 2.12 (i,vi) that $S \neq S_{2 n}(q)$ and by Lemma 2.12 (ii) that $(L, S) \neq\left(O_{2 n+1}(q), O_{2 n}^{-}(q)\right)$. Also $(L, S) \neq$ $\left(S_{2 n}(q), O_{2 n+1}(q)\right)$ by Lemma 3.3. Therefore $L \in\left\{O_{2 n+1}(q), S_{2 n}(q)\right\}$ and $S=O_{2 n}^{-}(q)$. If $n=4$, the proof is complete.

Suppose that $n \geqslant 5$. Let $s=r_{2 n-2}(q)$. Then $s$ divides the order of a parabolic subgroup of $S$ with Levi factor of type ${ }^{2} D_{n-1}$ and $p s \notin \omega(S)$ by Lemma 2.8. So $S$ has a Frobenius subgroup $F$ whose kernel is a $p$-group and whose complement has order $s$. For every $n$ we will find $r, w \in \pi(L)$ such that $r \in \pi(K)$ and $\{s, r, w\}$ is a coclique in $G K(L)$, and then will apply Lemma 3.4 to the group $F$ and the primes $r$ and $w$.

Let $n$ be odd and let $r=r_{n m}(p)$. Since $r \in \pi(L) \backslash \pi(S)$ and $r \notin \pi($ Out $S$ ) by Lemma 3.2, it follows that $r \in \pi(K)$. Exploiting Lemma 3.5, we deduce that $\left\{s, r, r_{2 n}(q)\right\}$ is a coclique in $G K(L)$ : both $r$ and $s$ do not divide $q^{n}+1$, and $r$ does not divide $q^{n-1}+1$ nor $q \pm 1$.

Let $n$ be even. Let $r_{1}=r_{(n-2) m}(p), r_{2}=r_{(n+2) m}(p)$ if $(n, q) \neq(8,2)$ and $r_{1}=r_{3}(2)$, $r_{2}=r_{5}(2)$ otherwise. Then $r_{1} r_{2} \in \omega(L) \backslash \omega(S)$ by Lemmas 2.7, 2.6 and 2.9, Furthermore, $r_{1}, r_{2} \notin \pi($ Out $S)$ by Lemma 3.2. Therefore at least one of $r_{1}$ and $r_{2}$ divides $|K|$. Denote this number by $r$. Since $n \geqslant 6$, both $n+2$ and $n-2$ do not divide $2 n-2$, and so $r$ is not adjacent to $s$ nor $r_{n-1}(q)$ in $G K(L)$ by Lemma 3.5. By the same lemma, $s$ and $r_{n-1}(q)$ are not adjacent either, and $\left\{s, r, r_{n-1}(q)\right\}$ is the desired coclique.

Applying Lemma 3.4, we conclude that $p t \in \omega(G)$ for all $t \in \pi(S), t \neq p$. But $r_{2 n}(q) \in$ $\pi(S)$ and $\operatorname{pr}_{2 n}(q) \notin \omega(L)$ by Lemmas 2.6 and 2.7, a contradiction.

Lemma 3.9. If $L=O_{2 n}^{+}(q)$, where $n \geqslant 6$ is even, then $S \notin\left\{S_{2 n-2}(q), O_{2 n-1}(q)\right\}$.

Proof. Assume the contrary. Let $s=r_{n-1}(q)$ and $w=r_{2 n-2}(q)$. Then $s$ divides the order of a parabolic subgroup of $S$ with Levi factor of type $A_{n-2}$ and $p s \notin \omega(S)$, therefore, $S$ contains a Frobenius group whose kernel is a $p$-group and whose complement has order $s$. Furthermore, Lemma 3.6 implies that $s$ and $w$ are not adjacent in $G K(L)$. Let $r_{1}=r_{(n-2) m}(p), r_{2}=$ $r_{(n+2) m}(p)$ if $(n, q) \neq(8,2)$, and $r_{1}=r_{3}(2), r_{2}=r_{5}(2)$ otherwise. Then $r_{1} r_{2} \in \omega(L) \backslash \omega(S)$ by Lemmas 2.7, 2.6] and 2.9, and $r_{1}, r_{2} \notin \pi($ Out $S)$ by Lemma 3.2. Thus at least one of the numbers $r_{1}$ and $r_{2}$ divides $|K|$. Denote this number by $r$. Both $n+2$ and $n-2$ do not divide $2 n-2$, and so $\{s, r, w\}$ is a coclique in $G K(L)$ by Lemma 3.6. By Lemma 3.4, we have $\operatorname{pr}_{2 n-2}(q) \in \omega(G)$. On the other hand, $p r_{2 n-2}(q) \notin \omega(L)$ by Lemma 2.8, a contradiction.

We have considered all the cases in the conclusion of Lemma 3.1, and so the proof of Theorem 2 is complete.

\section{NeW EXAMPles OF NON-QUASIRECOGNIZABle SIMPLE GROUPS}

A finite nonabelian simple group $L$ is said to be quasirecognizable by spectrum if every finite group isospectral to $L$ has only one nonabelian composition factor and this factor is isomorphic to $L$. Clearly quasirecognizability is a necessary condition for being almost recognizable. 
Recall that for even $q$, the simple group $S_{2 n}(q)$ is equal to $S p_{2 n}(q)$ and the simple group $O_{2 n}^{\varepsilon}(q)$ is $\Omega_{2 n}^{\varepsilon}(q)$, a subgroup of index 2 in $G O_{2 n}^{\varepsilon}(q)$ (see, for example, [7, p. xii]). Mazurov and Moghaddamfar [28] noted that $\omega\left(S p_{8}(2)\right)=\omega\left(G O_{8}^{-}(2)\right)$, and thereby $S p_{8}(2)$ is not quasirecognizable by spectrum. We generalize this result to all even $q$.

Proposition 4.1. Let $q$ be even. Then $\omega\left(S p_{8}(q)\right)=\omega\left(G O_{8}^{-}(q)\right)$ and in particular $S p_{8}(q)$ is not quasirecognizable by spectrum.

Proof. Denote $S p_{8}(q), G O_{8}^{-}(q)$, and $\Omega_{8}^{-}(q)$ by $L, G$, and $S$ respectively. Since $G$ is a subgroup in $G O_{9}(q) \simeq S p_{8}(q)$, it follows that $\omega(G) \subseteq \omega(L)$. Thus it suffices to show that $\omega(L) \backslash \omega(S) \subseteq$ $\omega(G)$. It follows from Lemma 2.7 that $\omega(L)$ consists of all divisors of the numbers $q^{4} \pm 1$, $\left(q^{2} \pm q+1\right)\left(q^{2}-1\right), 2\left(q^{3} \pm 1\right), 2\left(q^{2}+1\right)(q \pm 1), 4\left(q^{2} \pm 1\right)$, and $8(q \pm 1)$. Supplementing this by Lemma 2.11, we see that every number in $\omega(L) \backslash \omega(S)$ is a divisor of one of the numbers $2\left(q^{3} \pm 1\right), 4\left(q^{2}+1\right)$, and $8(q \pm 1)$.

It is well known that $G O_{2 n}^{-}(q)$ has a subgroup of the form $G O_{2 k}^{\varepsilon}(q) \times G O_{2 n-2 k}^{-\varepsilon}(q)$ for every $1 \leqslant k \leqslant n-1$ and $\varepsilon \in\{+,-\}$. Thus $G$ contains $G O_{6}^{+}(q) \times G O_{2}^{-}(q), G O_{4}^{+}(q) \times G O_{4}^{-}(q)$, and $\mathrm{GO}_{2}^{+}(q) \times G O_{6}^{-}(q)$.

It can be verified by means of [10] that $4 \in \omega\left(G O_{4}^{+}(2)\right)$. Since $G O_{4}^{+}(2) \leqslant G O_{4}^{+}(q)$ and $S L_{2}\left(q^{2}\right)<G O_{4}^{-}(q)$, it follows that $4\left(q^{2}+1\right) \in \omega(G)$.

Since $q^{3}-\varepsilon \in \omega\left(G O_{6}^{\varepsilon}(q)\right)$ and $G O_{2}^{\varepsilon}(q)$ is dihedral of order $2(q-\varepsilon)$, we conclude that $2\left(q^{3} \pm 1\right) \in \omega(G)$. Furthermore, $S_{8} \simeq G O_{6}^{+}(2) \leqslant G O_{6}^{+}(q)$, and hence $8(q+1) \in \omega(G)$. It remains to establish that $G O_{6}^{-}(q)$ has an element of order 8 .

The group $G O_{6}^{-}(q)$ is isomorphic to a split extension of $S U_{4}(q)$ by a graph automorphism of order 2. We identify $S U_{4}(q)$ with

$$
H=\left\{A \in S L_{4}\left(q^{2}\right) \mid A J \bar{A}^{\top}=J\right\},
$$

where $\overline{\left(a_{i j}\right)}=\left(a_{i j}^{q}\right)$ and $J$ is the $4 \times 4$ matrix with 1's on the antidiagonal and 0's elsewhere. Then $\gamma$ defined by $A^{\gamma}=\bar{A}$ is a graph automorphism of $H$. Choose $t \in G F\left(q^{2}\right)$ such that $t^{q} \neq t$ and let

$$
B=\left(\begin{array}{cccc}
1 & t & 0 & 0 \\
0 & 1 & 1 & 0 \\
0 & 0 & 1 & t^{q} \\
0 & 0 & 0 & 1
\end{array}\right)
$$

It is easy to verify that $B \in H$ and

$$
(B \gamma)^{4}=\left(\begin{array}{cccc}
1 & 0 & 0 & t^{2}+t^{2 q} \\
0 & 1 & 0 & 0 \\
0 & 0 & 1 & 0 \\
0 & 0 & 0 & 1
\end{array}\right)
$$

Since $t^{2}+t^{2 q} \neq 0$, the order of $B \gamma$ is equal to 8 , and hence $8 \in \omega\left(G O_{6}^{-}(q)\right)$.

It is worth noting that another example of a finite group isospectral to $S_{8}(q)$ with $q$ even and having $O_{8}^{-}(q)$ as a composition factor was constructed in [17].

Acknowledgments. We are grateful to the referee for his careful reading of the article and helpful comments and suggestions. 


\section{REFERENCES}

[1] M. R. Aleeva, On finite simple groups with the set of element orders as in a Frobenius group or a double Frobenius group, Math. Notes 73 (2003), no. 3, 299-313.

[2] O. A. Alekseeva and A. S. Kondrat'ev, On recognizability of some finite simple orthogonal groups by spectrum, Proc. Steklov Inst. Math. 266 (2009), no. suppl. 1, S10-S23.

[3] R. Brandl and W.J. Shi, Finite groups whose element orders are consecutive integers, J. Algebra 143 (1991), no. 2, 388-400.

[4] R. Brandl and W.J. Shi, The characterization of PSL(2,q) by its element orders, J. Algebra 163 (1994), no. 1, 109-114.

[5] A. A. Buturlakin, Spectra of finite symplectic and orthogonal groups, Siberian Adv. Math. 21 (2011), no. $3,176-210$.

[6] A. A. Buturlakin and M. A. Grechkoseeva, The cyclic structure of maximal tori of the finite classical groups, Algebra Logic 46 (2007), no. 2, 73-89.

[7] J. H. Conway, R. T. Curtis, S. P. Norton, R. A. Parker, and R. A. Wilson, Atlas of finite groups, Clarendon Press, Oxford, 1985.

[8] M. Foroudi Ghasemabadi, A. Iranmanesh, and N. Ahanjideh, Characterizations of the simple group ${ }^{2} D_{n}(3)$ by prime graph and spectrum, Monatsh. Math. 168 (2012), no. 3-4, 347-361.

[9] M. Foroudi Ghasemabadi, A. Iranmanesh, and N. Ahanjideh, 2-recognizability of the simple groups $B_{n}(3)$ and $C_{n}(3)$ by prime graph, Bull. Iranian Math. Soc. 39 (2013), no. 6, 1273-1281.

[10] The GAP Group, GAP - Groups, Algorithms, and Programming, Version 4.7.5, 2014.

[11] D. Gorenstein, R. Lyons, and R. Solomon, The classification of the finite simple groups. Number 3, Mathematical Surveys and Monographs, vol. 40.3, American Mathematical Society, Providence, RI, 1998.

[12] I. B. Gorshkov, Recognizability of alternating groups by spectrum, Algebra Logic 52 (2013), no. 1, $41-45$.

[13] M. A. Grechkoseeva, On element orders in covers of finite simple classical groups, J. Algebra 339 (2011), 304-319.

[14] M. A. Grechkoseeva, On spectra of covers of finite simple classical groups, Dokl. Math. 84 (2011), no. 1, $464-466$.

[15] M. A. Grechkoseeva, On element orders in covers of finite simple groups of Lie type, J. Algebra Appl. 14 (2015), 1550056 [16 pages].

[16] M. A. Grechkoseeva and D. V. Lytkin, Almost recognizability by spectrum of finite simple linear groups of prime dimension, Siberian Math. J. 53 (2012), no. 4, 645-655.

[17] M. A. Grechkoseeva and A. M. Staroletov, Unrecognizability by spectrum of finite simple orthogonal groups of dimension nine, Sib. Élektron. Mat. Izv. 11 (2014), 921-928.

[18] P. Hall and G. Higman, On the $p$-length of $p$-soluble groups and reduction theorem for Burnside's problem, Proc. London Math. Soc. 6 (1956), no. 3, 1-42.

[19] H. He and W. Shi, A note on the adjacency criterion for the prime graph and characterization of $C_{p}(3)$, Algebra Colloq. 19 (2012), no. 3, 553-562.

[20] H. He and W. J. Shi, Recognition of some finite simple groups of type $D_{n}(q)$ by spectrum, Int. J. Algebra Comput. 19 (2009), no. 5, 681-698.

[21] P. Kleidman and M. Liebeck, The subgroup structure of the finite classical groups, London Mathematical Society Lecture Note Series, vol. 129, Cambridge University Press, Cambridge, 1990.

[22] A. S. Kondrat'ev, On prime graph components of finite simple groups, Math. USSR-Sb. 67 (1990), no. $1,235-247$.

[23] A. S. Kondrat'ev, Recognition by spectrum of the groups ${ }^{2} D_{2^{m}+1}(3)$, Sci. China Ser. A 52 (2009), no. 2, 293-300.

[24] V. D. Mazurov, Characterizations of finite groups by sets of orders of their elements, Algebra and Logic 36 (1997), no. 1, 23-32.

[25] V. D. Mazurov, Recognition of finite groups by a set of orders of their elements, Algebra and Logic 37 (1998), no. 6, 371-379. 
[26] V. D. Mazurov, Recognition of finite simple groups $S_{4}(q)$ by their element orders, Algebra Logic 41 (2002), no. 2, 93-110.

[27] V. D. Mazurov, Unrecognizability by spectrum for a finite simple group ${ }^{3} D_{4}(2)$, Algebra Logic 52 (2013), no. 5, 400-403.

[28] V. D. Mazurov and A. R. Moghaddamfar, The recognition of the simple group $S_{8}(2)$ by its spectrum, Algebra Colloq. 13 (2006), no. 4, 643-646.

[29] V. D. Mazurov and W.J. Shi, A note to the characterization of sporadic simple groups, Algebra Colloq. 5 (1998), no. 3, 285-288.

[30] V. D. Mazurov, M.C. Xu, and H.P. Cao, Recognition of the finite simple groups $L_{3}\left(2^{m}\right)$ and $U_{3}\left(2^{m}\right)$ by their element orders, Algebra and Logic 39 (2000), no. 5, 324-334.

[31] R. Richardson, G. Röhrle, and R. Steinberg, Parabolic subgroups with Abelian unipotent radical, Invent. Math. 110 (1992), no. 3, 649-671.

[32] R. Shen, W.J. Shi, and M. R. Zinov'eva, Recognition of simple groups $B_{p}(3)$ by the set of element orders, Siberian Math. J. 51 (2010), no. 2, 244-254.

[33] W.J. Shi, The characterization of the sporadic simple groups by their element orders, Algebra Colloq. 1 (1994), no. 2, 159-166.

[34] W.J. Shi and C.Y. Tang, A characterization of some orthogonal groups, Progr. Natur. Sci. 7 (1997), no. $2,155-162$.

[35] A. M. Staroletov, On recognition by spectrum of the simple groups $B_{3}(q), C_{3}(q)$ and $D_{4}(q)$, Siberian Math. J. 53 (2012), no. 3, 532-538.

[36] A. V. Vasil'ev, On connection between the structure of a finite group and the properties of its prime graph, Siberian Math. J. 46 (2005), no. 3, 396-404.

[37] A. V. Vasil'ev, On finite groups isospectral to simple classical groups, J. Algebra 423 (2015), 318-374.

[38] A. V. Vasil'ev and I. B. Gorshkov, On recognition of finite simple groups with connected prime graph, Siberian Math. J. 50 (2009), no. 2, 233-238.

[39] A. V. Vasil'ev, I. B. Gorshkov, M. A. Grechkoseeva, A. S. Kondrat'ev, and A. M. Staroletov, On recognizability by spectrum of finite simple groups of types $B_{n}, C_{n}$, and ${ }^{2} D_{n}$ for $n=2^{k}$, Proc. Steklov Inst. Math. 267 (2009), no. suppl. 1, 218-233.

[40] A. V. Vasil'ev and M. A. Grechkoseeva, Recognition by spectrum for finite simple linear groups of small dimensions over fields of characteristic 2, Algebra Logic 47 (2008), no. 5, 314-320.

[41] A. V. Vasil'ev, M. A. Grechkoseeva, and V. D. Mazurov, On finite groups isospectral to simple symplectic and orthogonal groups, Siberian Math. J. 50 (2009), no. 6, 965-981.

[42] A. V. Vasil'ev and A. M. Staroletov, Almost recognizability of simple exceptional groups of Lie type, Algebra Logic 53 (2015), no. 6, 433-449.

[43] A. V. Vasil'ev and E.P. Vdovin, An adjacency criterion for the prime graph of a finite simple group, Algebra Logic 44 (2005), no. 6, 381-406.

[44] A. V. Vasil'ev and E. P. Vdovin, Cocliques of maximal size in the prime graph of a finite simple group, Algebra Logic 50 (2011), no. 4, 291-322.

[45] J. S. Williams, Prime graph components of finite groups, J. Algebra 69 (1981), 487-513.

[46] A. V. Zavarnitsine, Recognition of the simple groups $L_{3}(q)$ by element orders, J. Group Theory 7 (2004), no. $1,81-97$.

[47] A. V. Zavarnitsine, Recognition of the simple groups $U_{3}(q)$ by element orders, Algebra Logic 45 (2006), no. $2,106-116$.

[48] A. V. Zavarnitsine, Exceptional action of the simple groups $L_{4}(q)$ in the defining characteristic, Sib. Élektron. Mat. Izv. 5 (2008), 65-74.

[49] K. Zsigmondy, Zur Theorie der Potenzreste, Monatsh. Math. Phys. 3 (1892), 265-284. 